\title{
Implementación de un manojo de medidas (bundle) de inserción para prevenir la infección del torrente sanguíneo asociada a dispositivo intravascular central en Cuidado Intensivo en Colombia
}

\author{
Johanna Osorio, Diana Álvarez, Robinson Pacheco, Carlos A. Gómez y Abner Lozano
}

\section{Implementation of an insertion bundle for preventing central line-associated bloodstream infections in an Intensive Care Unit in Colombia}

Background: Central line-associated bloodstream infections (CLABSI) related to insertion and device care in intensive care units are frequent and preventable events. Aim: To evaluate the reduction in the rate of CLABSI through implementation of an insertion bundle. Methods: A study was conducted in the Adult-ICU at the University Hospital of Neiva comparing a pre-interventional period with an interventional one, each lasting 6 months; the intervention consisting of implementing a bundle of measures for the insertion of central venous catheters (CVC). In the pre-intervention period (2010) the rate of CLABSI and the population's characteristics were evaluated. The bundle for the insertion of the CVC consisted in: hands hygiene, use of $2 \%$ clorhexidine, maximum sterile barriers and avoiding femoral access. Results: The rate of CLABSI decreased from 5.56 to 3.26 per 1000 catheter days. The length of ICU stay and catheter duration were associated with a higher risk of infection associated to these devices $(\mathrm{p}<0.05)$. Compliance with the bundle is a protective factor against the development of CLABSI (OR $0.45, p=0.615$ ). The staff adherence to the bundle was over $80 \%$. Conclusion: Implementing a Central Line Insertion Bundle proved to be a useful measure in prevention of CLABSI in our hospital. This strategy could be implemented in other hospitals of similar complexity.

Key words: Vascular access devices, central line associated bloodstream infection, catheter-related infection, bundle, risk factors.

Palabras clave: Accesos vasculares, infección del torrente sanguíneo asociada a catéter, infección asociada a dispositivo intravascular, manojo de medidas, factores de riesgo.

\section{Introducción}

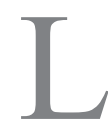

as infecciones asociadas a la atención en salud (IAAS) tradicionalmente han sido consideradas como eventos adversos inherentes a la atención en salud. Sin embargo, la implementación de procesos relacionados con la seguridad del paciente y la calidad en la prestación de los servicios han cambiado a lo largo del tiempo esta concepción, clasificándolas en eventos prevenibles, cuyos indicadores cada vez deben ser más cercanos a cero $^{1-4}$.

La neumonía asociada a ventilación mecánica (NAVM), la infección del sitio operatorio y la infección del torrente sanguíneo asociada a dispositivos intravasculares, entre otras, son condiciones frecuentemente observadas, que se presentan en 5 a $10 \%$ de los pacientes hospitalizados. Puntualmente la infección asociada a dispositivos intravasculares centrales representa un alto impacto en morbilidad, tiempo de estancia y costos de la hospitalización, siendo éste cercano a los \$50.000 USD para cada episodio $^{5,6}$. Por estas razones, todo esfuerzo encaminado a la prevención de las IAAS, especialmente en países de medianos y bajos ingresos, como Colombia, tiene una importancia y utilidad incuestionable ${ }^{7}$.

La literatura científica reporta que la mortalidad por todas las causas en las unidades de cuidado intensivo (UCI) en Colombia es de $31 \%$, la cual está ligada a la gravedad de la enfermedad, edad y co-morbilidades ${ }^{8}$. Para este tipo de población, los dispositivos intravasculares, entre ellos los catéteres venosos centrales (CVC) son ampliamente empleados para monitoreo hemodinámico, soporte vasopresor, nutricional e infusión de medicamentos, entre otros. Sin embargo, el uso de estos dispositivos también trae consigo complicaciones asociadas a su presencia y manipulación, como son las infecciosas, trombóticas y mecánicas 9 .

El riesgo de infección del torrente sanguíneo varía de acuerdo al tipo de dispositivo intravascular, el tiempo de uso del mismo, la experiencia de la persona que lo inserta, la frecuencia de uso del catéter, las características del
Hospital Universitario de Neiva, Colombia (JO, DA, AL). Centro Internacional de Investigaciones y Entrenamiento Médico (CIDEIM). Cali, Colombia (RP). University of Pittsburgh Medical Center. Pennsylvania, E.U.A. (CAG)

Establecimiento donde se realizó el trabajo: Hospital Universitario de Neiva, Colombia.

Los autores declaran no tener conflictos de interés Financiamiento: no hubo

Recibido: 31 de agosto de 2012 Aceptado: 6 de agosto de 2013

Correspondencia a: Johanna Osorio P. osoriopinzon@gmail.com 
paciente cateterizado y el uso de estrategias preventivas basadas en la higiene de manos y uso de métodos de barrera estériles para la inserción y manipulación del dispositivo, principalmente ${ }^{10}$. Se conoce que pacientes con presencia de co-morbilidades como diabetes mellitus, enfermedad renal crónica, infección por VIH/SIDA, malignidad hematológica, tumor sólido o algún otro tipo de inmunosupresión, tienen una probabilidad mayor de presentar este tipo de complicaciones ${ }^{4,11-13}$.

En diversos países, se ha introducido recientemente la aplicación de manojos de medidas (en inglés bundles) soportadas en la evidencia científica, para la prevención de las infecciones intrahospitalarias relacionadas con dispositivos o con exposiciones determinadas ${ }^{14,15}$. Estos manojos de medidas se han empleado en la prevención de $\mathrm{NAVM}^{16-23}$, infección del sitio operatorio ${ }^{24}$, infección del tracto urinario ${ }^{25,26}$, infecciones por microorganismos resistentes como Staphylococcus aureus y Acinetobacter baumannii ${ }^{27} \mathrm{y}$, en menor proporción, en la prevención de la infección del torrente sanguíneo asociada a dispositivos intravasculares ${ }^{28-34}$, logrando una reducción de hasta $54 \%$ en las tasas ajustadas de infección asociadas a catéter ${ }^{32}$. Incluso, podrían implementarse protocolos de este tipo para mejorar la prescripción de antimicrobianos en los hospitales $^{35,36}$.

El Hospital Universitario de la ciudad de Neiva (HUN), Colombia, es una institución pública de tercer nivel de complejidad, centro de referencia del Departamento del Huila, que tiene una población aproximada de 1 millón de habitantes. El HUN tiene 400 camas, y cuenta con una UCI de 21 camas para la atención de pacientes adultos con patologías médico-quirúrgicas. En esta UCI, la tasa de infección asociada a catéter venoso central (IACVC) ha estado, durante los últimos cinco años, por encima de $6 \times 1.000$ días de exposición al dispositivo, por lo que se le consideró como un sitio clave en el cual implementar una estrategia preventiva.

Adicionalmente, el HUN cuenta con un sistema de vigilancia epidemiológica activa desde el año 2007, conformado por el mismo equipo de trabajo hasta la fecha, el cual recolecta, concentra y revisa la información correspondiente a las infecciones asociadas a la atención en salud, entre ellas las del torrente sanguíneo.

\section{Objetivo}

Determinar la efectividad de la aplicación de la estrategia de manojo de medidas para la reducción de infección asociada a catéter intravascular central en la UCI del Hospital Universitario de Neiva Colombia.

\section{Materiales y Métodos}

\section{Estudio de intervención tipo antes y después}

- Primer período (Período 1): Comprendido entre 1 de abril de 2010 y 30 de septiembre de 2010, en el que se calculó la tasa de IACVC teniendo en cuenta los criterios de definición propuestos por $\mathrm{CDC} / \mathrm{NHSN}^{37}$ y los días de exposición a dispositivo intravascular central. Adicionalmente, se evaluaron las características de la población admitida a la UCI adultos del HUN durante este período.

- Segundo período (Período 2): Entre 1 de abril de 2011 y 30 de septiembre de 2011, que consiste en la aplicación del manojo de medidas para la prevención de las infecciones asociadas a dispositivos intravasculares centrales, supervisión de su cumplimiento bajo pauta de cotejo por parte del equipo encargado de la atención del paciente en el momento de requerir un acceso vascular central, recolección de la información necesaria para el cálculo de la tasa ajustada de infección por catéter central, seguimiento de la adherencia a la higienización de manos y retroalimentación de los resultados al personal asistencial.

Las medidas basadas en la evidencia más importantes que configuraron el manojo de medidas para prevención de infecciones por catéteres vasculares centrales fueron: el lavado o higienización de manos antes y después del contacto con el paciente siguiendo la técnica recomendada por la OMS, el uso de máximas barreras estériles durante la inserción del catéter central, la preparación de la piel del paciente con clorhexidina al $2 \%$ y evitar el acceso vascular femoral ${ }^{11,12,29-34}$. Adicionalmente, se revisó diariamente con el grupo tratante la necesidad de permanencia del catéter en cada paciente.

\section{Población}

Individuos hospitalizados en UCI adultos en los dos períodos señalados, con indicación de inserción de CVC, que cumplieran con:

- Criterios de inclusión: Individuos mayores de 18 años hospitalizados en la UCI del HUN con indicación de acceso venoso central con dispositivo no tunelizado, de acuerdo a criterio del médico tratante, siempre y cuando fueran insertados dentro de la UCI o en el quirófano (según el estado clínico del paciente): Se considera al quirófano un ambiente adecuado para realizar procedimientos invasores bajo condiciones óptimas de asepsia y antisepsia, haciéndolo equiparable al manojo de medidas.

- Criterios de exclusión: Individuos remitidos de otras instituciones o procedentes de otro servicio de hospitalización con CVC. Individuos con indicación de línea arterial, catéter para hemodiálisis o catéter central de inserción periférica; así como aquellos que desarrollaran infección del torrente sanguíneo en las primeras $48 \mathrm{~h}$ de hospitalización. 
El equipo de control de infecciones hospitalarias del HUN revisó cada caso sospechoso de infección del torrente sanguíneo asociado a dispositivo intravascular, empleando los criterios propuestos por el CDC/NHSN para la clasificación de dicha patología ${ }^{37}$.

De acuerdo a la información suministrada por el laboratorio de microbiología sobre hemocultivos positivos se realizó la búsqueda adicional de posibles casos.

Se escogió los dos períodos de tiempo similares (abril a septiembre de 2010 y de 2011) teniendo en cuenta diversos factores como cambios de personal, festividades, período de vacaciones, etc., que pudieran afectar los resultados del estudio. Es importante aclarar que la única intervención adicional que se realizó en el hospital durante el período de estudio fue la elaboración e implementación de las guías de manejo antimicrobiano de la institución, a partir de junio de 2011.

Se elaboró una pauta de cotejo inicial, la cual se empleó como prueba piloto en algunos participantes no incluidos en el segundo período del estudio (antes del 1 de abril de 2011), con posteriores ajustes para lograr incluir en ella la información necesaria para el adecuado procesamiento y análisis de los datos.

Se realizó el cálculo de la tasa de infecciones asociadas a dispositivo intravascular central por 1.000 días de catéter. En pacientes con más de una infección por catéter sólo se consideró el primer episodio y no se contaron los días de catéter posteriores a dicho episodio para efectos del análisis. Adicionalmente, se tuvieron en cuenta las variables clínicas, socio-demográficas, los días de exposición a CVC en los dos períodos y la mortalidad de los pacientes al egreso de UCI.

El desarrollo del estudio fue aprobado por el Comité de Ética del HUN, el Comité de Infecciones y el Comité de Docencia e Investigación del HUN, teniendo en cuenta que comprendía medidas basadas en la evidencia científica con alta probabilidad de obtener resultados positivos y que no contemplaba otras intervenciones que se apartaran de la práctica clínica habitual.

\section{Análisis estadístico}

Los análisis se realizaron con el software estadístico Stata 12. Todas las variables se describieron según su característica; las variables categóricas con medidas de frecuencia y las variables continuas con proporciones, medidas de tendencia central y de dispersión. La comparación de variables categóricas se realizó con $\chi^{2}$ o test exacto de Fisher cuando fue apropiado. Para evaluar la distribución de las poblaciones se aplicó la prueba de normalidad de Kolmogorov Smirnov. Posteriormente se empleó en primera instancia un análisis univariado, con $\mathrm{T}$ test para muestras independientes, $\chi^{2} \mathrm{o}$ el Test exacto de Fisher en los casos correspondientes. Se calcularon las tasas de IACVC y se compararon por medio de la regresión de Poisson. Para la significancia estadística se consideró un valor de $\mathrm{p}<0,05$ y los intervalos de confianza de $95 \%$.

\section{Resultados}

En la UCI de adultos hubo una exposición a CVC de 2.112 días durante el período 1. Para el período 2, este dato correspondió a 1.833 días de CVC.

Ingresaron al estudio 579 participantes que cumplieron con los criterios de elegibilidad, 279 en el período 1 y 285 en el período 2. No se logró obtener información completa de la historia clínica o del formulario de recolección en 15 de ellos, dos en el período de intervención y 13 en el pre-intervención (no se encontraron datos sobre comorbilidades o tipo de admisión a la UCI) por lo que se excluyeron.

No hubo reportes de infecciones del torrente sanguíneo ocurridas en las primeras $48 \mathrm{~h}$ de la admisión a UCI en ninguno de los dos períodos.

Las características demográficas de los participantes durante los dos períodos se resumen en la Tabla 1, en la que se observa que no hubo diferencias significativas entre los dos grupos en edad, sexo, tipo de admisión, días de estancia, días de exposición a CVC, por lo que las poblaciones se consideraron comparables. En cuanto a las co-morbilidades, se encontró que existían diferencias con significancia estadística en patologías como diabetes mellitus y enfermedad renal, las cuales, sin embargo, se presentaron en mayor proporción en el período 2 .

La mediana de edad en el período 1 fue de 55 años y de 50 para el período 2. En los dos períodos, predominó el sexo masculino y la causa de ingreso más frecuente a UCI fue quirúrgica. Los días de exposición al CVC por paciente fueron similares en los dos grupos (mediana de 4) y la mortalidad observada se acercó a $30 \%$ en los dos períodos.

Las tasas de IACVC correspondientes al período 1 y 2 fueron de 5,56 y 3,26 casos por 1.000 días de CVC respectivamente, resaltándose una reducción en la tasa de IACVC de 41,36\% con la aplicación del manojo de medidas. Expresado en forma de razón, se tuvo una relación de 1,7:1 infecciones relacionadas con el dispositivo intravascular en los períodos 1 y 2 , respectivamente. El número de infecciones asociadas a CVC fue de 9 en el período 1 y de 5 en el período 2, con lo cual se redujeron los casos de IACVC en 44,4\%, tras la intervención.

En el segundo período, se documentaron dos episodios de IACVC de los insertados en el quirófano, comparado con tres casos de IACVC de la UCI, con un promedio de 11 y 8,5 días de permanencia del CVC previo al desarrollo de infección, respectivamente.

Durante el período 2, se aplicó un manojo de medidas 
Tabla 1. Características generales de la población de UCI - abril a septiembre de 2010 y 2011

\begin{tabular}{|c|c|c|c|c|c|}
\hline Característica & Descripción & $\begin{array}{c}\text { Período } 1 \\
\text { (antes de la intervención) } \\
n=279\end{array}$ & $\begin{array}{c}\text { Período } 2 \\
\text { (aplicación de manojo de medidas) } \\
n=285\end{array}$ & $\begin{array}{l}\text { Tipo de } \\
\text { prueba }\end{array}$ & Valor $p$ \\
\hline Edad en años & $\begin{array}{l}\text { Mediana } \\
\text { Rango intercuartílico }\end{array}$ & $\begin{array}{r}55 \\
37-70\end{array}$ & $\begin{array}{r}50 \\
36-71\end{array}$ & $\mathrm{~T}$ test & 0,9918 \\
\hline Sexo & $\begin{array}{l}\mathrm{n}(\%) \text { masculino } \\
\mathrm{n}(\%) \text { femenino }\end{array}$ & $\begin{array}{r}187(67,03) \\
92(32,97)\end{array}$ & $\begin{array}{r}204(71,58) \\
81(28,42)\end{array}$ & $\chi^{2}$ & 0,241 \\
\hline Tipo de admisión UCl & $\begin{array}{l}\text { n (\%) médico } \\
\text { n (\%) quirúrgico } \\
\text { n (\%) traumático }\end{array}$ & $\begin{array}{r}95(34,05) \\
109(39,07) \\
75(26,88)\end{array}$ & $\begin{array}{r}89(31,23) \\
111(38,95) \\
85(29,82)\end{array}$ & $\chi^{2}$ & 0,7751 \\
\hline 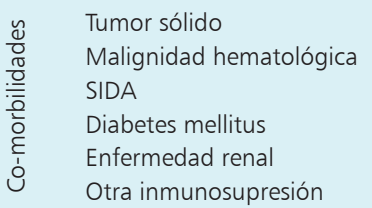 & $\begin{array}{l}\text { n (\%) } \\
\text { n (\%) } \\
\text { n (\%) } \\
\text { n (\%) } \\
\text { n (\%) } \\
\text { n (\%) }\end{array}$ & $\begin{aligned} 25 & (8,96) \\
1 & (0,36) \\
2 & (0,72) \\
26 & (9,32) \\
15 & (5,38) \\
8 & (2,87)\end{aligned}$ & $\begin{array}{rr}17 & (5,96) \\
5 & (1,75) \\
6 & (2,11) \\
44 & (15,44) \\
28 & (9,82) \\
4 & (1,40)\end{array}$ & $\begin{array}{l}\chi^{2} \\
\text { Fisher } \\
\text { Fisher } \\
\chi^{2} \\
\chi^{2} \\
\text { Fisher }\end{array}$ & $\begin{array}{l}0,175 \\
0,216 \\
0,286 \\
0,028 \\
0,047 \\
0,257\end{array}$ \\
\hline Mortalidad al egreso de UCl & $n(\%)$ & $72(25,81)$ & $80(28,07)$ & $\chi^{2}$ & 0,545 \\
\hline Días de exposición a CVC & $\begin{array}{l}\text { Mediana } \\
\text { Rango intercuartílico }\end{array}$ & $\begin{array}{r}4 \\
2-8\end{array}$ & $\begin{array}{r}4 \\
2-7\end{array}$ & T test & 0,3201 \\
\hline Días de estancia en UCl & $\begin{array}{l}\text { Mediana } \\
\text { Rango intercuartílico }\end{array}$ & $\begin{array}{r}6 \\
4-11\end{array}$ & $\begin{array}{r}6 \\
3-12\end{array}$ & $T$ test & 0,5185 \\
\hline
\end{tabular}

Tabla 2. Aplicación del manojo de medidas - abril a septiembre de 2011

\begin{tabular}{|c|c|c|c|}
\hline \multicolumn{2}{|l|}{ Característica } & Descripción & $\begin{array}{l}\text { Período } 2 \text { (aplicación del } \\
\text { manojo de medidas) } \\
n=285\end{array}$ \\
\hline \multicolumn{2}{|c|}{$\begin{array}{l}\text { Cumplimiento del manojo de medidas } \\
\text { Catéteres insertados en UCl }\end{array}$} & n (\%) Sí & $106 / 131(80,91)$ \\
\hline \multirow{2}{*}{\multicolumn{2}{|c|}{ Sitio de inserción CVC }} & $\mathrm{n}(\%) \cup \mathrm{Cl}$ & $131(45,96)$ \\
\hline & & n (\%) quirófano & $154(54,04)$ \\
\hline \multirow{3}{*}{\multicolumn{2}{|c|}{ Ubicación del CVC }} & n (\%) subclavio & $125(43,86)$ \\
\hline & & n (\%) yugular & $158(55,44)$ \\
\hline & & n (\%) femoral & $2(0,70)$ \\
\hline \multirow{4}{*}{$\begin{array}{l}\text { Pasos del manojo } \\
\text { de medidas }\end{array}$} & Lavado de manos & n (\%) Sí & $260(91,23)$ \\
\hline & Uso de clorhexidina & n (\%) Sí & $260(91,23)$ \\
\hline & Máximas barreras & n (\%) Sí & $259(90,88)$ \\
\hline & Evitar acceso femoral & n (\%) Sí & $259(90,88)$ \\
\hline \multicolumn{2}{|c|}{ Cumplimiento global de medidas preventivas } & n (\%) Sí & $258(90,53)$ \\
\hline \multirow{5}{*}{\multicolumn{2}{|c|}{ Número de punciones }} & n (\%) una & $70(24,56)$ \\
\hline & & n (\%) dos & $17(5,96)$ \\
\hline & & $\mathrm{n}(\%)$ tres & $9(3,16)$ \\
\hline & & n (\%) cuatro & $12(4,21)$ \\
\hline & & $\mathrm{n}(\%)$ desconocido & $177(62,11)$ \\
\hline
\end{tabular}

consistente en la implementación de condiciones asépticas para la inserción de catéteres centrales en $90,53 \%$ de los procedimientos realizados tanto en el quirófano como en UCI (Tabla 2). No se encontraron diferencias en adherencia a cada medida contemplada en el manojo de medidas cuando éstas se evaluaron en forma individual. En el período 2 no se diligenció la pauta de cotejo en 25 procedimientos de inserción de CVC ocurridos en la UCI, los cuales se asumieron como no adherencia a la medida de intervención. Entre los $131 \mathrm{CVC}$ insertados en la UCI, hubo cumplimiento del manojo de medidas en $80,91 \%$ de los casos.

No fue posible evaluar la destreza del personal que insertó el dispositivo o el grado de dificultad del procedimiento con relación al número de punciones necesarias, dado que en una importante proporción de pacientes, los catéteres se insertaron en quirófano y no quedó consignado el registro en la historia clínica o en el formulario de recolección de datos.

Aunque no fue posible determinar el número de CVC insertados en el quirófano durante el año 2010, es probable que en ese año se realizara un número similar de procedimientos a los observados durante 2011, ya que el tipo de ingreso quirúrgico que facilitara que el catéter fuera insertado en el quirófano fue similar en los dos períodos.

En la Tabla 3 se puede apreciar un mayor número de días de permanencia del CVC y un exceso de días de 
estancia en UCI dos a tres veces mayor en los pacientes que desarrollaron IACVC frente a los que no lo hicieron, durante los dos períodos.

Todos los pacientes que presentaron infección asociada a catéter, la desarrollaron después del séptimo día de permanencia del CVC. En la Tabla 4 se resumen los episodios de IACVC ocurridos en ambos períodos. En cuanto a las co-morbilidades de los pacientes que cursaron con IACVC se encontraron tres casos de tumor sólido durante el año 2010 comparado con ninguna co-morbilidad evidenciada en el 2011.

De la Tabla 5 se desprende que el tiempo en días de estancia en UCI y los días de exposición al dispositivo intravascular incrementan el riesgo de desarrollar infecciones asociadas a CVC $(\mathrm{p}<0,05)$. En contraposición, el cumplimiento del manojo de medidas es un factor protector para desarrollar una IACVC $(\mathrm{OR}=0,45 ; \mathrm{p}=0,615)$.

Adicionalmente, al realizar la comparación de las tasas de infección asociada a CVC por 1.000 días de exposición al dispositivo en los dos períodos, se observó que la oportunidad de adquirir un episodio de IACVC si se emplea un manojo de medidas, es 0,58 veces la oportunidad de adquirirla si no se emplea este manojo de medidas, constituyéndose éste como factor protector, aunque no alcanzó significancia estadística $(\mathrm{p}=0,339$, IC 0,19-1,75).

\section{Discusión}

Los resultados obtenidos en este estudio sugieren que la implementación de un manojo de medidas puede ser una herramienta ampliamente aceptada entre el personal de salud, contando con adherencia superior a $80 \%$, comparada con $38 \%$ reportado por Furuya y cols. ${ }^{12} \mathrm{y}$, adicionalmente demuestra que existe un $42 \%$ de oportunidad para disminuir los episodios de IACVC, tras su implementación.

Nuestros resultados sugieren una asociación entre los días de permanencia del CVC y los episodios de infección relacionada con el dispositivo. Únicamente los pacientes que permanecieron con el catéter por más de siete días desarrollaron infección del torrente sanguíneo, a pesar de que el tiempo de permanencia del catéter previo a la infección en nuestro estudio fue inferior al reportado por otros autores, como Galpen ${ }^{31}$.

Una de las fortalezas de este estudio radica en el decidido intento de controlar los sesgos. Los sesgos de selección se minimizaron debido a que los sujetos participantes cumplieron a cabalidad con los criterios de inclusión propuestos. Del mismo modo, a pesar de contemplarse un diseño "antes y después" (períodos 1 y 2 , respectivamente), se puede afirmar que las dos poblaciones eran comparables en relación a sexo, edad, tipo de
Tabla 3. Comparación días CVC y estancia en UCI de pacientes con y sin IACVC-abril a septiembre de 2010 y 2011

\begin{tabular}{|c|c|c|c|c|}
\hline \multirow[b]{2}{*}{ IACVC } & \multicolumn{2}{|c|}{2010} & \multicolumn{2}{|c|}{2011} \\
\hline & Sí & No & Sí & No \\
\hline $\begin{array}{l}\text { Días de CVC } \\
\text { Total (promedio) }\end{array}$ & $119(13,2)$ & $1.498(5,54)$ & $54(10,8)$ & $1.477(5,2)$ \\
\hline $\begin{array}{l}\text { Días de estancia en UCl } \\
\text { Total (promedio) }\end{array}$ & $164(18,2)$ & $2.198(8,11)$ & $133(26,6)$ & $2.411(8,6)$ \\
\hline
\end{tabular}

Tabla 4. Comparación de episodios de IACVC en la UCI - abril y septiembre de 2010 y 2011

\begin{tabular}{|c|c|c|c|}
\hline Característica & Descripción & $\begin{array}{c}\text { Período } 1 \\
\text { (antes de la } \\
\text { intervención) } \\
n=9\end{array}$ & $\begin{array}{c}\text { Período } 2 \\
\text { (aplicación del } \\
\text { manojo de medidas) } \\
n=5\end{array}$ \\
\hline $\begin{array}{l}\text { Tipo de microorganismo } \\
\text { aislado }\end{array}$ & $\begin{array}{l}\mathrm{n}(\%) P \text {. aeruginosa } \\
\mathrm{n}(\%) \text { E. cloacae } \\
\mathrm{n}(\%) \text { S. aureus } \\
\mathrm{n}(\%) \text { K. pneumoniae } \\
\mathrm{n}(\%) \text { A. baumannii } \\
\mathrm{n}(\%) \text { S. coagulasa negativa } \\
\mathrm{n}(\%) \text { Otros }\end{array}$ & $\begin{array}{l}2(22,2) \\
2(22,2) \\
1(11,1) \\
1(11,1) \\
0(0) \\
1(11,1) \\
2(22,2)\end{array}$ & $\begin{array}{l}1(20) \\
0(0) \\
1(20) \\
0(0) \\
1(20) \\
0(0) \\
2(40)\end{array}$ \\
\hline Sexo & $\begin{array}{l}\text { n (\%) Hombres } \\
n(\%) \text { Mujeres }\end{array}$ & $\begin{array}{l}7(77,7) \\
2(22,2)\end{array}$ & $\begin{array}{l}4(80) \\
1(20)\end{array}$ \\
\hline Mortalidad & n (\%) Sí & $6(66,6)$ & $0(0)$ \\
\hline Tipo de admisión a UCI & $\begin{array}{l}\text { n (\%) Médica } \\
\text { n (\%) Quirúrgica } \\
\text { n (\%) Traumática }\end{array}$ & $\begin{array}{l}4(44,4) \\
3(33,3) \\
2(22,2)\end{array}$ & $\begin{array}{c}0(0) \\
2(40) \\
3(60)\end{array}$ \\
\hline 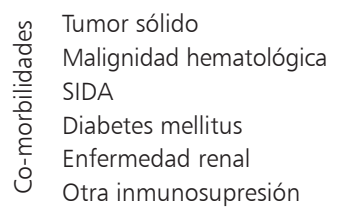 & $\begin{array}{l}\text { n (\%) Sí } \\
\text { n (\%) Sí } \\
n(\%) \text { Sí } \\
n(\%) \text { Sí } \\
n(\%) \text { Sí } \\
n(\%) \text { Sí }\end{array}$ & $\begin{array}{l}3(33,33) \\
0(0) \\
0(0) \\
0(0) \\
0(0) \\
0(0)\end{array}$ & $\begin{array}{l}0(0) \\
0(0) \\
0(0) \\
0(0) \\
0(0) \\
0(0)\end{array}$ \\
\hline Días CVC & $\begin{array}{l}\text { Mediana } \\
\text { Rango intercuartílico }\end{array}$ & $\begin{array}{c}11 \\
8-17\end{array}$ & $\begin{array}{c}9 \\
8-10\end{array}$ \\
\hline Días estancia & $\begin{array}{l}\text { Mediana } \\
\text { Rango intercuartílico }\end{array}$ & $\begin{array}{c}20 \\
10-22\end{array}$ & $\begin{array}{c}15 \\
10-30\end{array}$ \\
\hline
\end{tabular}

UCI: unidad de cuidado intensivo. CVC: catéter venoso central. IACVC: infección asociada a catéter venoso central.

Tabla 5. Análisis multivariado-IACVC relacionada con días de CVC y días de estancia UCl-2010 y 2011

\begin{tabular}{|clccc}
$\begin{array}{c}\text { Período de } \\
\text { seguimiento }\end{array}$ & Variables a medir & $\begin{array}{c}\text { Odds } \\
\text { ratio }\end{array}$ & $\mathbf{p}$ & IC 95\% \\
\hline 2010 & IACVC/días CVC & 1,14 & 0,000 & $1,06-1,22$ \\
& IACVC/días de estancia en UCI & 1,09 & 0,001 & $1,03-1,15$ \\
2011 & IACVC/días CVC & 1,11 & 0,030 & $1,01-1,22$ \\
& IACVC/días de estancia en UCI & 1,08 & 0,001 & $1,03-1,13$ \\
& IACVC/se cumplió el manojo de medidas & 0,45 & 0,615 & $0,20-9,84$ \\
\hline
\end{tabular}

UCI: unidad de cuidado intensivo. CVC: catéter venoso central. IACVC: infección asociada a catéter venoso central. 
admisión y, en general las co-morbilidades, exceptuando diabetes mellitus y enfermedad renal, las cuales fueron incluso más frecuentes en el período de intervención. Los posibles sesgos de información se controlaron empleando una prueba piloto en la cual se evalúo el instrumento; adicionalmente se revisó la historia clínica del paciente con el fin de cotejar la información y se verificó la base de datos elaborada por parte de los dos investigadores. A pesar de lo anterior, no se lograron obtener los datos completos de la historia clínica en 15 pacientes que cumplían con los criterios de inclusión; no obstante, este porcentaje de pérdidas fue menor a $3 \%$ y no afectó en gran medida los resultados obtenidos, dado que se verificó con el equipo de vigilancia epidemiológica del hospital y ninguno de estos pacientes desarrolló IACVC durante su estancia hospitalaria.

Es importante tener en cuenta que, si bien este tipo de diseño trae consigo grandes limitaciones (entre ellos la no existencia de un grupo control simultáneo que disminuya la posibilidad de sesgos y que los resultados puedan deberse al azar), hay consideraciones muy importantes, entre las cuales se resaltan las éticas, que impiden hacer esta comparación paralela, dado que se podría estar atentando contra la seguridad del paciente, ofreciendo a un grupo las mejores prácticas basadas en la evidencia y al otro la práctica clínica usual; por lo que se prefirió el abordaje "antes y después".

Pese a estas limitaciones, en la institución no han ocurrido cambios en el número de camas de la UCI, no se ha modificado la razón personal asistencial: paciente, o los proveedores de insumos, ni se han implementado, adicionalmente a la vigilancia epidemiológica (existente en la institución como un proceso definido desde antes de 2010), medidas específicas dirigidas a la prevención de infecciones diferentes al manojo de medidas, por lo que no encontramos otra razón evidente que pueda explicar el descenso de las tasas de IACVC en el período de intervención.

También se tuvieron en cuenta factores que pudiesen confundir como las co-morbilidades o tipo de admisión a UCI que pudieran haber influido en los resultados, como es el caso de diabetes mellitus y enfermedad renal, patologías que se presentaron en mayor proporción durante el período de la intervención y que, como lo plantean algunos autores, se constituyen en factores de riesgo para el desarrollo de la infección del torrente sanguíneo asociada a $\mathrm{CVC}^{13}$. A pesar de este escenario clínico adverso para el segundo período, se obtuvo una reducción de $44 \%$ de las infecciones asociadas a dispositivo intravascular durante la intervención, resaltando el impacto de la instauración del manojo de medidas.

Otra fortaleza fue el haber contemplado períodos de tiempo similares, tanto en duración como en los meses de observación e intervención (abril a septiembre de
2010 y 2011, respectivamente) con lo cual se redujo la posibilidad de que los resultados obtenidos en el segundo período se explicaran por la presencia de factores externos como cambio de personal laboral o de entrenamiento, festividades o factores estacionales.

En cuanto a la mortalidad por todas las causas en los dos períodos de estudio, no se encontraron diferencias significativas. Sin embargo, en los pacientes que presentaron IACVC se documentó una mortalidad de 66,6\% en el período pre intervención, comparada con 0 durante el período 2. Aunque este cambio brusco no puede ser atribuido a la instauración del manojo de medidas, ya que se trata de una estrategia preventiva, hay que mencionar que a comienzos de junio de 2011 se implementaron en el HUN las guías de uso de antimicrobianos, que pudieron contribuir a una disminución en la mortalidad de los pacientes con IACVC, al proporcionar un antimicrobiano empírico más apropiado, de acuerdo al perfil microbiológico institucional. Sin embargo, el inicio del presente estudio también pudo haber motivado cambios en el comportamiento del personal de salud que favoreciera la adherencia tanto al protocolo como a las guías de uso de antimicrobiano en la UCI. Vale la pena resaltar que dichas guías no tuvieron influencia en el desarrollo de los episodios de IACVC per se, dado que no se emplean rutinariamente antimicrobianos en la prevención de infecciones del torrente sanguíneo o como profilaxis previa al procedimiento de inserción de dispositivos intravasculares.

Durante el período 2 se presentó una reducción en el número de días de permanencia del CVC sin poder determinar una clara explicación para este fenómeno. Sin embargo, podría pensarse que una disminución en el denominador del indicador tasa de IACVC podría incrementar el resultado de dicho indicador, lo cual no se observó en nuestro estudio, dado que hay una clara disminución de la tasa de IACVC en el período de intervención.

Nuestros resultados muestran un exceso de 18 días de estancia en los pacientes que desarrollaron IACVC en el período 2. Dado que este estudio fue desarrollado en un país de medianos ingresos (Colombia), es importante resaltar que medidas con relativo bajo costo y de alto impacto en la reducción de complicaciones infecciosas asociadas a la atención en salud, como este manojo de medidas, requieren una implementación prioritaria; en contraposición a lo planteado por Apisarnthanarak y cols. ${ }^{32}$, quienes emplearon medidas adicionales a la implementación del manojo de medidas, como cambio de la cobertura del CVC cada 48 h o 7 días, dependiendo del material utilizado. En el presente estudio no se modificó medida alguna relacionada con el cuidado y manipulación del catéter, lo cual también puede tener un impacto en la disminución de las tasas de IACVC a expensas del incremento en los costos de la atención. 
En el estudio desarrollado por Pronovost y cols. ${ }^{28}$, se observó una disminución en la tasa de incidencia de IACVC desde 1 episodio por 1.000 días de exposición al dispositivo a 0,76 por 1.000 días catéter durante el período de intervención, sin resultados iniciales que tuvieran significancia estadística, los cuales fueron similares a los documentados por nosotros. Sin embargo, Pronovost, hecho el análisis a los 18 meses, documentó que la tasa de incidencia de IACVC había disminuido aún más, $(0,34$ por 1.000 días de exposición a CVC), hallazgos esta vez con significancia estadística y que plantean la necesidad de hacer estudios de duración más prolongada.

En nuestro estudio, con posterioridad a la implementación del manojo de medidas, se evidenció que la oportunidad de adquirir un episodio de IACVC fue 0,58 veces la oportunidad de adquirirla si no se empleara. Dicho resultado, a pesar de su gran relevancia clínica, no contó con significancia estadística y se puede deber al número reducido de IACVC documentadas en los dos períodos $\mathrm{y}$, a su vez, al tiempo de evaluación relativamente corto (seis meses para cada uno), lo cual en un comienzo refleja una falencia a tener en cuenta para futuros estudios.

Aunque se trata del primer trabajo de intervención que se desarrolló en el HUN para la prevención de IAAS, se documentó tras esta intervención una reducción cercana a $45 \%$ de los episodios de IACVC, lo cual plantea un impacto positivo de la implementación del manojo de medidas en la prevención de infecciones del torrente sanguíneo. Se hace necesario proponer a futuro un estudio más prolongado, idealmente multicéntrico, en instituciones de nivel de complejidad similar, que evalúe el impacto de la estrategia a gran escala.

No obstante y debido a que es una intervención soportada con medidas basadas en la evidencia científica, que no pone en riesgo la seguridad en la atención de los pacientes y que a muy bajo costo otorga un importante beneficio, se podría extender dicha práctica a toda la población que requiera un CVC.

\section{Conclusiones}

La seguridad de los pacientes cobra cada día mayor relevancia en las instituciones de salud y la prevención de las IAAS es la piedra angular para el ejercicio de los equipos de control de infecciones, los cuales tienen la misión de prevenir y vigilar la aparición de dichos eventos adversos en los pacientes que se hospitalizan. El empleo de manojo de medidas para la inserción de líneas centrales tiene un impacto positivo en la disminución de complicaciones infecciosas derivadas de dichos procedimientos, incluso en pacientes con diabetes mellitus y enfermedad renal. Es importante resaltar que en instituciones hospitalarias de complejidad similar, sobre todo en países de medianos ingresos, el manojo de medidas constituye una buena alternativa para la prevención de infecciones asociadas al uso de dispositivos intravasculares, con relativo bajo costo y mínimo riesgo para el paciente.

Agradecimientos: A Esperanza García, microbióloga del HUN y al personal de la UCI por su disposición y colaboración para el desarrollo del presente estudio.

\section{Resumen}

Introducción: Las infecciones asociadas a la instalación y manejo de catéteres venosos centrales (CVC) son eventos frecuentes en unidades de cuidados intensivos pero evitables. Objetivo: Evaluar la eficacia en disminuir la tasa de infección asociada a catéter (IACVC) obtenida con la implementación de un manojo de medidas (bundle) durante la inserción del dispositivo. Material y Métodos: Se condujo un estudio que compara un período preintervención con uno de intervención, de 6 meses cada uno, consistente en la implementación de un manojo de medidas para la inserción de catéter venoso central (CVC), en la Unidad de Cuidado Intensivo (UCI) del Hospital Universitario de Neiva, Colombia. En el período pre-intervención (2010) se evaluó la tasa de IACVC y las características de la población. Durante la intervención (2011) se implementó un manojo de medidas para la inserción de CVC que consistió en: higiene de manos, uso de clorhexidina $2 \%$, empleo de máximas barreras estériles y evitar el acceso femoral. Resultados: Se obtuvo reducción de la tasa de IACVC de 5,56 a 3,26 X 1.000 días CVC. Los días de estancia en UCI y de exposición al $\mathrm{CVC}$ se asociaron a mayor riesgo de desarrollar IACVC $(\mathrm{p}<0,05)$; el cumplimiento del manojo de medidas fue un factor protector contra IACVC (OR 0,$45 ; \mathrm{p}=0,615)$. La adherencia del personal al manojo de medidas fue mayor de $80 \%$. Conclusión: La implementación de un manojo de medidas para la inserción de CVC resultó ser una medida útil para la prevención de IACVC en nuestro hospital, lo que podría implementarse en otras instituciones hospitalarias de complejidad similar. 


\section{Referencias bibliográficas}

1.- Marra A R, Cal R G, Durão M S, Correa L, Guastelli L R, Moura D F, et al. Impact of a program to prevent central line-associated bloodstream infection in the zero tolerance era. Am J Infect Control 2010; 38 (6): 434-9.

2.- Berriel-Cass D, Adkins F W, Jones P, Fakih M. Eliminating nosocomial infections at ascension health. Jt Comm J Qual Patient Saf 2006; 32 (11): 612-20.

3.- McLaws M L, Burrell A R. Zero risk for central line-associated bloodstream infection: are we there yet? Crit Care Med 2012; 40 (2): 388-93.

4.- Zingg W, Walder B, Pittet D. Prevention of catheter-related infection: toward zero risk? Curr Opin Infect Dis 2011; 24 (4): 377-84.

5.- Casey A L, Elliott T S. Prevention of central venous catheter-related infection: update. Br J Nurs 2010; 19 (2): 78-82.

6.- Miller R S, Norris P R, Jenkins J M, Talbot T R 3rd, Starmer J M, Hutchison S A, et al. Systems initiatives reduce healthcareassociated infections: a study of 22,928 device days in a single trauma unit. J Trauma 2010; 68 (1): 23-31.

7.- Chittick P, Sherertz R J. Recognition and prevention of nosocomial vascular device and related bloodstream infections in the intensive care unit. Crit Care Med 2010; 38v (Suppl 8): S363-S372.

8.- Dennis R J, Pérez A, Rowan K, Londoño D, Metcalfe A, Gómez C, et al. Factores asociados con la mortalidad hospitalaria en pacientes admitidos en cuidados intensivos en Colombia. Arch Bronconeumol 2002; 38 (3): 117-22.

9.- $\quad$ McGee D, Gould M. Preventing complications of central venous catheterization. N Engl J Med 2003; 348: 1123-33.

10.- O'Grady N P, Alexander M, Burns L A, Dellinger E P, Garland J, Heard S O, et al. Guidelines for the prevention of intravascular catheter-related infections. Clin Infect Dis 2011; 52 (9): e162-e93.

11.- Render M L, Brungs S, Kotagal U, Nicholson M, Burns P, Ellis D, et al. Evidence-based practice to reduce central line infections. Jt Comm J Qual Patient Saf 2006; 32: 253-60.

12.- Furuya E Y, Dick A, Perencevich E N, Pogorzelska M, Goldmann D, Stone P W. Central line bundle implementation in US intensive care units and impact on bloodstream infections. PLoS One 2011; 18; 6 (1): e15452.

13.- Erbay A, Ergönül O, Stoddard G J, Samore M H. Recurrent catheter-related bloodstream infections: risk factors and outcome. Int J Infect Dis 2006; 10: 396-400.

14.- Venkatram S, Rachmale S, Kanna B. Study of device use adjusted rates in healthcareassociated infections after implementation of "bundles" in a closed-model medical intensive care unit. J Crit Care 2010; 25 (1): 174. e11-18.

15.- Bonello R S, Fletcher C E, Becker W K, Clutter K L, Arjes S L, Cook J J, et al. An intensive care unit quality improvement collaborative in nine Departments of Veterans Affairs hospitals: reducing ventilator-associated pneumonia and catheter-related bloodstream infection rates. Jt Comm J Qual Patient Saf 2008; 34 (11): 639-45.

16.- Bird D, Zambuto A, O’Donnell C, Silva J, Korn C, Burke R, et al. Adherence to ventilatorassociated pneumonia bundle and incidence of ventilator-associated pneumonia in the surgical intensive care unit. Arch Surg 2010; 145 (5): 465-70.

17.- Rello J, Lode H, Cornaglia G, Masterton R. VAP Care Bundle Contributors. A European care bundle for prevention of ventilatorassociated pneumonia. Intensive Care Med 2010; 36 (5): 773-80.

18.- O'Brien P J, Joss J A, Crofts S L. Application of a ventilator care bundle and outcome. Br J Anaesth 2010; 104 (1): 112.

19.- Zilberberg M D, Shorr A F, Kollef M H. Implementing quality improvements in the intensive care unit: ventilator bundle as an example. Crit Care Med 2009; 37 (1): 305-9.

20.- Blamoun J, Alfakir M, Rella M E, Wojcik J M, Solis R A, Anees Khan M, et al. Efficacy of an expanded ventilator bundle for the reduction of ventilator-associated pneumonia in the medical intensive care unit. Am J Infect Control 2009; 37 (2): 172-5.

21.- O'Keefe-McCarthy S, Santiago C, Lau G. Ventilator-associated pneumonia bundled strategies: an evidence-based practice. Worldviews Evid Based Nurs 2008; 5 (4): 193-204.

22.- Westwell S. Implementing a ventilator care bundle in an adult intensive care unit. Nurs Crit Care 2008; 13 (4): 203-7.

23.- Tolentino-De los Reyes A F, Ruppert S D, Shiao S Y. Evidence-based practice: use of the ventilator bundle to prevent ventilatorassociated pneumonia. Am J Crit Care 2007; 16 (1): 20-7.

24.- Awad S S, Palacio C H, Subramanian A, Byers P A, Abraham P, Lewis D A, et al. Implementation of a methicillin-resistant Staphylococcus aureus (MRSA) prevention bundle results in decreased MRSA surgical site infections. Am J Surg 2009; 198 (5): 607-10.

25.- Winter M, Helms B, Harrington L, Luquire R, McVay T, Rhodes N. Eliminating catheterassociated urinary tract infections: part I. Avoid catheter use. J Health Qual 2009; 31 (6): 8-12.

26.- Saint S, Olmsted R N, Fakih M G, Kowalski C P, Watson S R, Sales A E, et al. Translating health care-associated urinary tract infection prevention research into practice via the bladder bundle. Jt Comm J Qual Patient Saf 2009; 35 (9): 449-55.

27.- Rodríguez-Baño J, García L, Ramírez E, Martínez-Martínez L, Muniain M A, Fernández-Cuenca F, et al. Long-term control of hospital-wide, endemic multidrugresistant Acinetobacter baumannii through a comprehensive "bundle" approach. Am J Infect Control 2009; 37 (9): 715-22.

28.- Pronovost P, Needham D, Berenholtz S, Sinopoli D, Chu H, Cosgrove S, et al. An intervention to decrease catheter-related bloodstream infections in the ICU. N Engl J Med 2006; 355: 2725-32.

29.- Smith M. A care bundle for management of central venous catheters. Paediatr Nurs 2007; 19 (4): 39-44.

30.- Harnage S. Innovative bundle wipes out catheter-related bloodstream infections. Nursing 2008; 38 (10): 17-8.

31.- Galpern D, Guerrero A, Tu A, Fahoum B, Wise L. Effectiveness of a central line bundle campaign on line-associated infections in the intensive care unit. Surgery 2008; 144 (4): 492-5.

32.- Apisarnthanarak A, Thongphubeth $\mathrm{K}$, Yuekyen C, Warren D K, Fraser V J. Effectiveness of a catheter-associated bloodstream infection bundle in a Thai tertiary care center: a 3-year study. Am J Infect Control 2010; 38 (6): 449-55.

33.- Royer T. Implementing a better bundle to achieve and sustain a zero central lineassociated bloodstream infection rate. J Infus Nurs 2010; 33 (6): 398-406. 
34.- Kim J, Holtom P, Vigen C. Reduction of catheter-related bloodstream infections through the use of a central venous line bundle: Epidemiologic and economic consequences. Am J Infect Control 2011; 39: 640-6.

35.- Pulcini C, Defres S, Aggarwal I, Nathwani D, Davey P. Design of a 'day 3 bundle' to improve the reassessment of inpatient empirical antibiotic prescriptions. J Antimicrob Chemother 2008; 61 (6): 1384-8.

36.- Cooke F J, Holmes A H. The missing care bundle: antibiotic prescribing in hospitals. Int J Antimicrob Agents 2007; 30 (1): 25-9.
37.- CDC/NHSN Surveillance Definition of Healthcare-Associated Infection and Criteria for Specific Types of Infections in the Acute Care Setting. January 2012. www.cdc.gov/nhsn/pdfs/pscmanual/ 17pscnosinfdef_current.pdf. (accedido 29 de julio de 2012). 